\title{
Tristetraprolin: A novel target of diallyl disulfide that inhibits the progression of breast cancer
}

\author{
TING XIONG $^{1 *}$, XIAO-WANG LIU ${ }^{1 *}$, XUE-LONG HUANG $^{1}$, XIONG-FENG XU ${ }^{1}$, \\ WEI-QUAN XIE ${ }^{1}$, SU-JUN ZHANG ${ }^{2}$ and JIAN TU ${ }^{1}$ \\ ${ }^{1}$ Institute of Pharmacy and Pharmacology, \\ Hunan Province Cooperative Innovation Center for Molecular Target New Drug Study; \\ ${ }^{2}$ Experimental Animal Department, University of South China, Hengyang, Hunan 421001, P.R. China
}

Received August 15, 2017; Accepted February 9, 2018

DOI: $10.3892 / 01.2018 .8299$

\begin{abstract}
Diallyl disulfide (DADS), a volatile component of garlic oil, has various biological properties, including antioxidant, antiangiogenic and anticancer effects. The present study aimed to explore novel targets of DADS that may slow or stop the progression of breast cancer. First, xenograft tumor models were created by subcutaneously injecting MCF-7 and MDA-MB-231 breast cancer cells into nude mice. Subsequently, western blot analysis was performed to investigate the expression of tristetraprolin (TTP), urokinase-type plasminogen activator (uPA) and matrix metalloproteinase-9 (MMP-9) in the xenograft tumors, and cell cultures. Tablet cloning, Transwell and wound healing assays revealed that DADS treatment significantly inhibited the proliferation, invasion and migration of breast cancer cells. In addition, DADS treatment led to significant downregulation of uPA and MMP-9 protein expression, but significantly upregulated TTP expression in vivo and in vitro. Knocking down TTP expression using small interfering RNA reversed the aforementioned effects of DADS, which suggests TTP is a key target of DADS in inhibiting the progression of breast cancer.
\end{abstract}

Correspondence to: Professor Jian Tu, Institute of Pharmacy and Pharmacology, Hunan Province Cooperative Innovation Center for Molecular Target New Drug Study, University of South China, Road 28 Changsheng Street, Hengyang, Hunan 421001, P.R. China E-mail: tujian0734@aliyun.com

Mrs. Su-Jun Zhang, Experimental Animal Department, University of South China, Road 28 Changsheng Street, Hengyang, Hunan 421001, P.R. China

E-mail: 1248942982@qq.com

*Contributed equally

Abbreviations: DADS, diallyl disulfide; MMP, matrix metalloproteinase; uPA, urokinase-type plasminogen activator; AU, adenylate-uridylate; ARE, AU-rich element; TTP, tristetraprolin; siRNA, small interfering RNA

Key words: diallyl disulfide, breast cancer, tristetraprolin

\section{Introduction}

Breast cancer is the most common type of cancer that develops in women (1). Although the survival rate is high when diagnosed early, $\sim 50 \%$ of breast cancer-associated mortality is due to local invasion and distant metastasis $(2,3)$. Therefore, it is important to investigate the underlying molecular mechanisms that drive tumor formation and novel therapeutic strategies to target breast cancer.

Diallyl disulfide (DADS), one of the major volatile components of allicin, exhibits diverse anticancer activities, including cell cycle arrest, inhibition of cell growth, induction of cell differentiation and apoptosis by interfering with a variety of cell signaling pathways (4-8). However, the mechanism of DADS in breast cancer remains to be determined.

Numerous studies have revealed that diverse molecular mechanisms are implicated in the suppressive effects that DADS exhibit on tumor growth $(9,10)$. In particular, DADS has been demonstrated to decrease the invasiveness of gastric cancer cells by decreasing the expression and activity of matrix metalloproteinases (MMPs) (11). MMPs serve a key function in the tumor microenvironment by facilitating cell metastasis. MMP-9, a member of the MMP family, is known to be involved in tumor invasion and metastasis, and degrade the extracellular matrix in the vicinity of the tumor (12). In addition, urokinase-type plasminogen activator (uPA), one of the upstream genes of MMP-9, interacts with co-receptors to activate intracellular signaling pathways that mediate the degradation of the extracellular matrix, and activates plasminogen and MMPs (13). Increased expression levels of uPA and MMP-9 expression are indicators of poor prognosis in numerous human tumors, including breast cancer (14). In addition, adenylate-uridylate (AU)-rich elements (AREs) in the 3 -untranslated regions of numerous transiently expressed genes regulate mRNA, mediate post-transcriptional modifications and serve important functions in various types of cancer (15). Tristetraprolin (TTP) is an important AU-rich RNA-binding protein, which targets mRNAs (16). Previous studies have identified decreased expression of TTP in numerous types of cancer, including breast cancer, hepatocellular carcinoma, and head and neck cancer (17-19). TTP dysregulation has been implicated in the progress of various types of cancer, 
particularly in the proliferation, apoptosis, invasion, migration and resistance to chemotherapy of inflammation-associated types of cancer cells. Additionally, TTP may mediate the degradation of uPA mRNA, and inhibit the cell invasion and migration of tumors by altering the expression of MMP-9 (19). Therefore, the aim of the present study was to investigate the functions of DADS in the inhibition of cell invasion and metastasis of human breast cancer, which may be a beneficial therapy for this disease.

\section{Materials and methods}

Reagents and cell culture. DADS was purchased from Sigma-Aldrich; Merck KGaA (Darmstadt, Germany). The following antibodies were obtained: Rabbit anti-uPA primary antibody (cat. no. ABO10669; Abgent, Inc., San Diego, CA, USA); Rabbit anti-MMP-9 primary antibody (cat. no. ab73734; Abcam, Cambridge, UK); Mouse anti-TTP and mouse anti- $\beta$-actin primary antibodies (cat. no. SAB4200565 \& A1978; Sigma-Aldrich; Merck KgaA, Darmstadt, germany.); horseradish peroxidase (HRP)-conjugated goat anti-rabbit IgG and HRP-conjugated goat anti-mouse IgG secondary antibodies (cat. nos. 70-GAR0072 \& 70-GAM007; Wuhan Boster Biological Technology, Ltd., Wuhan, China). The bicinchoninic acid (BCA) protein assay and other reagents were obtained from Kangwei Biotechnology (CWBiotech, Beijing, China). Transwell ${ }^{\circledR}$ inserts were purchased from Corning Incorporated (Corning, NY, USA). MCF-7 and MDA-MB-231 human breast cancer cell lines were purchased from the Type Culture Collection of the Chinese Academy of Sciences (Shanghai, China). Cells were cultured in high-glucose Dulbecco's modified Eagle's medium (DMEM; Hyclone, Logan, UT, USA) supplemented with $10 \%$ fetal bovine serum (FBS; Gibco by Invitrogen, Carlsbad, CA, USA) at $37^{\circ} \mathrm{C}$ in a humidified atmosphere containing $5 \% \mathrm{CO}_{2}$.

In vivo tumorigenicity study. A total of 18 4-week-old female nude mice (weighing 16-18 g) were purchased from Shanghai SLAC Laboratory Animal Co., Ltd. (Shanghai, China). All mice were kept in a barrier facility under high-efficiency particulate air filtration and maintained in a specific pathogen-free environment with shavings as bedding. The cages and bedding were sterilized at $121^{\circ} \mathrm{C}$ for $20 \mathrm{~min}$. All mice were allowed free access to food and water, the feed was purchased from Ke'aoxieli Feed Co. Ltd (Peking, China), drinking water was sterilized by ${ }^{60} \mathrm{Co}$ irradiation. Approximately 1x107 MDA-MB-231 or MCF-7 cells were subcutaneously injected into the subcutis of the right axilla of each mouse. Tumor volume $\left(\mathrm{mm}^{3}\right)$ was examined every 4 days and calculated using a standard formula [width ${ }^{2} \mathrm{x}$ (length/2)]. When the tumor volume reached $80 \mathrm{~mm}^{3}$, the mice were divided into two groups. The control group used saline as vehicle treatment while the treatment group received $50 \mathrm{mg} / \mathrm{kg}$ DADS via intraperitoneal injection every 2 days. After 28 days, the xenografts were removed. The tumor weights were measured in both groups. All experimental procedures conformed to the Guide for the Care and Use of Laboratory Animals published by the US National Institutes of Health (NIH Publication No. 85-23, revised 1996) and were approved by the Institutional Animal Ethics Committee of University of South China (Hengyang, China).
Hematoxylin and eosin $(H \& E)$ staining. Tumor tissues (control group and DADS-treated group) were cut into $0.5-\mathrm{mm}^{3}$ sections, fixed using $4 \%$ paraformaldehyde for $24 \mathrm{~h}$ at room temperature, embedded in paraffin and further cut into $5 \mu \mathrm{m}$ thick tissue sections. The tissue sections were dewaxed in xylene and rehydrated in a descending (100, 95, 90, 80 and $75 \%$ ) ethanol series, 3-5 min each time. Following washing with PBS, the sections were stained with hematoxylin and following a second wash, the sections were differentiated. The sections were subsequently stained with eosin following washing. Following dehydration with ethanol, the sections were mounted, and observed using light with a x40 microscopy objective (Eclipse E200; Nikon Corporation, Tokyo, Japan).

Western blot analysis. The tumor tissues and cells were lysed using radioimmunoprecipitation assay buffer (Thermo Fisher Scientific, Inc., Waltham, MA, USA) supplemented with Halt Protease and Phosphatase Inhibitor Cocktail (1:100; Thermo Fisher Scientific, Inc.), sonicated and clarified by centrifugation at $12,000 \mathrm{x} \mathrm{g}, 15 \mathrm{~min}$ at $4^{\circ} \mathrm{C}$. All groups of protein were quantified using a bicinchoninic acid protein assay and equal amounts of total protein extracts $(30 \mu \mathrm{g} /$ well) were separated by $8-12 \%$ SDS-PAGE. Following electrophoresis for $2 \mathrm{~h}$ at $100 \mathrm{~V}$, the proteins were electrically transferred onto a polyvinylidene fluoride membrane. The membranes were then blocked with $5 \%$ nonfat dry milk for $1 \mathrm{~h}$ at room temperature and then incubated with primary antibodies against TTP (1:500), uPA $(1: 1,000)$, MMP-9 $(1: 1,000)$ or $\beta$-actin $(1: 1,000)$ at $4^{\circ} \mathrm{C}$ overnight. Thereafter, the membranes were washed three times with TBST $(20 \mathrm{mmol} / 1$ Tris base, $\mathrm{pH} 7.6,150 \mathrm{mmol} / \mathrm{l}$ $\mathrm{NaCl}$ and $0.1 \%$ Tween-20), incubated with the HRP-conjugated goat anti-rabbit or goat anti-mouse IgG secondary antibodies for $50 \mathrm{~min}$ at room temperature, and washed three times with TBST. Protein visualization was performed using the ECL Plus Western Blotting Detection system (Tanon-6200, Tanon Science \& Technology Co., Ltd., Shanghai, China) to collect the images and QuantityOne 4.5.0 software (Bio-Rad Laboratoris, Inc., Hercules, CA, USA) for analysis.

Tablet cloning assay. A total of 200/well MCF-7 and MDA-MB-231 cells were planted into $9 \mathrm{~cm}$ petri dishes. After treating cells with $0,100,200$ or $400 \mu \mathrm{M}$ DADS for $24 \mathrm{~h}$ (using $0 \mu \mathrm{M}$ as the control group), cells were washed with PBS three times, then fresh serum-free DMEM was added. After 3 weeks, $5 \mathrm{ml} 100 \%$ methanol was added to the dishes for $15 \mathrm{~min}$ to fix the cells at room temperature. Giemsa was used to stain cells for $20 \mathrm{~min}$ at room temperature. Following PBS washing of the cells and air-drying, the clone formation rate was calculated under microscope, clone formation rate $(\%)=($ clone number/plated cell number $) \times 100 \%$.

Transwell assays. For the Transwell assays, $25 \mu \mathrm{g}$ Matrigel was added to the upper side of porous filters (pore size, $8 \mu \mathrm{m}$ ) and the gel was allowed to form at $37^{\circ} \mathrm{C}$ for $2 \mathrm{~h}$. Following rehydration of the coated filters with $100 \mu \mathrm{l}$ medium, $1 \times 10^{6}$ cells in $100 \mu \mathrm{l}$ serum-free DMEM supplemented with $0.2 \%$ FBS were seeded into the upper part of each chamber, whereas the lower compartments were filled with $500 \mu \mathrm{l}$ complete DMEM containing $10 \%$ FBS to serve as the chemoattractant agent. Cells were incubated at $37^{\circ} \mathrm{C}$ with $5 \% \mathrm{CO}_{2}$ for $24 \mathrm{~h}$ to allow 

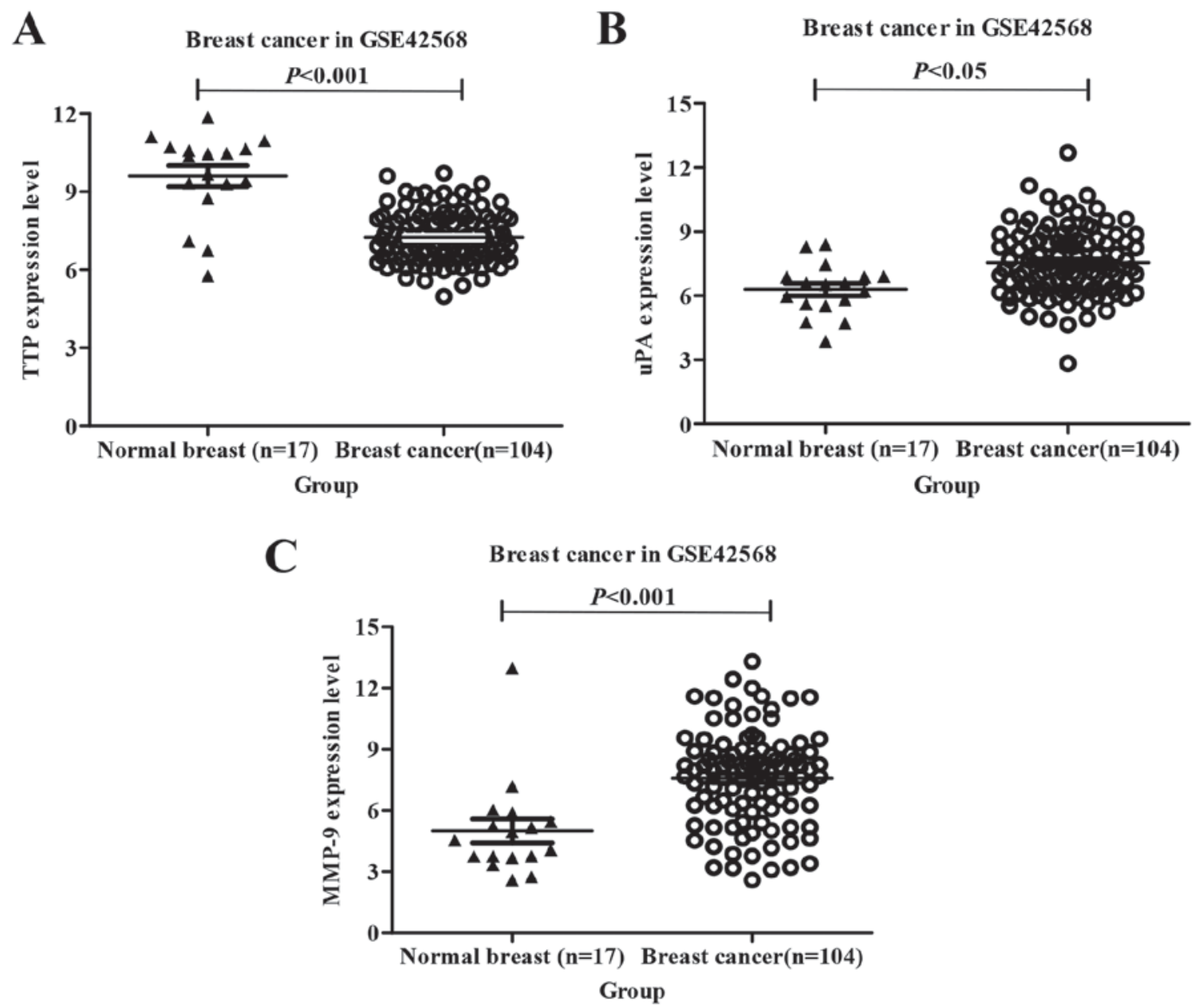

Figure 1. TTP mRNA expression is downregulated, whereas uPA and MMP-9 expression is upregulated in human breast cancer. The GSE42568 microarray dataset was downloaded from the Gene Expression Omnibus database, which compared breast cancer tissue with normal breast tissue. (A) TTP mRNA levels. (B) uPA mRNA levels. (C) MMP-9 mRNA levels. The database normalized TTP, uPA and MMP expression using the robust multiarray average method. MMP, matrix metalloproteinase; uPA, urokinase-type plasminogen activator; TTP, tristetraprolin.

for invasion. At $24 \mathrm{~h}$, cells on the upper surface of the filters were removed using cotton swabs. Cells that had invaded to the lower surface of the filter were washed twice with PBS, fixed with $4 \%$ paraformaldehyde for $15 \mathrm{~min}$ and stained with $0.1 \%$ crystal violet at room temperature. Images were captured using an epifluorescence inverted microscope (magnification, $\mathrm{x} 100$ ). The total number of invaded cells was normalized to the number of non-targeting control cells and expressed as fold change.

Wound healing assay. MCF-7 and MDA-MB-231 cells ( $2 \times 10^{5} /$ well) were cultured in 6 -well plates in DMEM containing $10 \%$ FBS. The cell monolayer in each well was scratched with a $200 \mu$ l plastic pipette tip to create a linear wound. The monolayer was washed twice with PBS to remove debris and detached cells, and the cells were then exposed to serum-free medium with or without various concentrations of DADS (100, 200 and $400 \mu \mathrm{M})$ for $24 \mathrm{~h}$ and using $0 \mu \mathrm{M}$ as control group. The wound areas were then observed using an inverted microscope (magnification, x100). The migration distance was measured, and migration rates are expressed as the ratio of the treated group value to the control group value.
Small interfering (si)RNA transfection. siRNA duplexes for TTP were designed and produced by Shanghai GenePharma Co., Ltd. (Shanghai, China). The sequences for TTP siRNA were sense, 5'-UCGCCACCCCAAGUACAAATT-3' and antisense, 5'-UUUGUAGGGGUGGCGATT-3'. Scramble control RNAi was used as control siRNA, the sequence for the negative control siRNA was 5'-GCAAGCTGACCC TGAAGTT-3'. The TTP and control siRNA were transfected into MCF-7 and MDA-MB-231 cells using Lipofectamine ${ }^{\circledR}$ 3000 (Invitrogen; Thermo Fisher Scientific, Inc.) according to the manufacturer's protocol. Briefly, MCF-7 and MDA-MB-231 cells (1×105/well) were inoculated into 6-well plates and cultured in DMEM with $10 \% \mathrm{FBS}$ at $37^{\circ} \mathrm{C}$ until reaching $70 \%$ confluence. Then, $2 \mu 1 \mathrm{TTP}$ or control siRNA $(0.5 \mu \mathrm{g} / \mu \mathrm{l})$ was mixed with $5 \mu \mathrm{l}$ Lipofectamine 3000 . The mixture was added to the cell culture, and cells were incubated for $12 \mathrm{~h}$ at $37^{\circ} \mathrm{C}$. The cell solution was replaced with DMEM containing $10 \%$ FBS and cells were incubated for another $24 \mathrm{~h}$.

Gene Expression Omnibus (GEO) database. The GSE42568 breast cancer microarray dataset was downloaded from the GEO database (https://www.ncbi.nlm.nih.gov/geo/). 
A

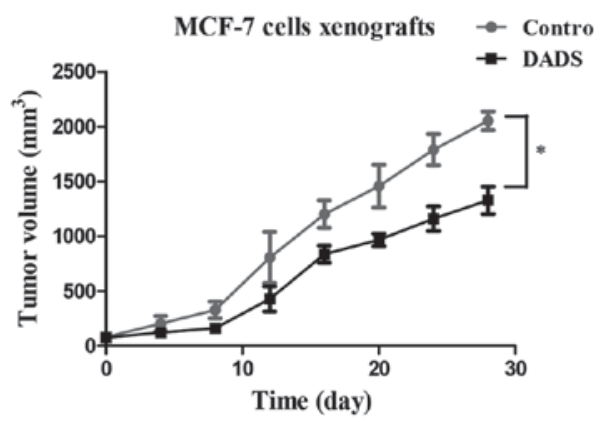

B

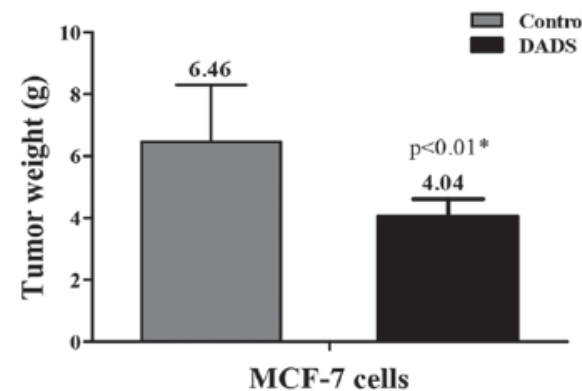

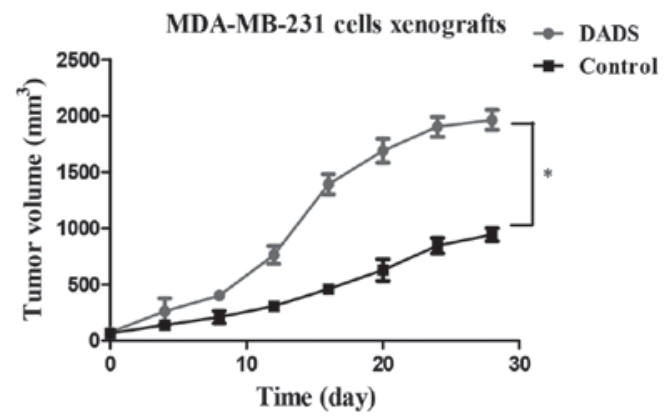

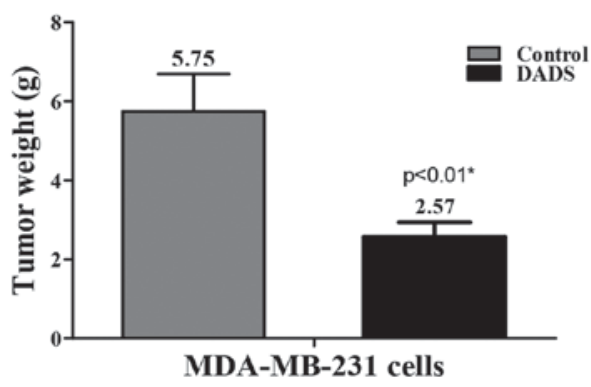

Figure 2. Tumor volume and tumor weight in the breast cancer xenografts. (A) Control vs. DADS treatment in tumor volume between days 0 and 28. (B) Control vs. DADS treatment in tumor weight on day 28 . Data are presented as the mean \pm standard deviation. ${ }^{*} \mathrm{P}<0.05$ vs. control; $\mathrm{n}=8$. DADS, diallyl disulfide.

A
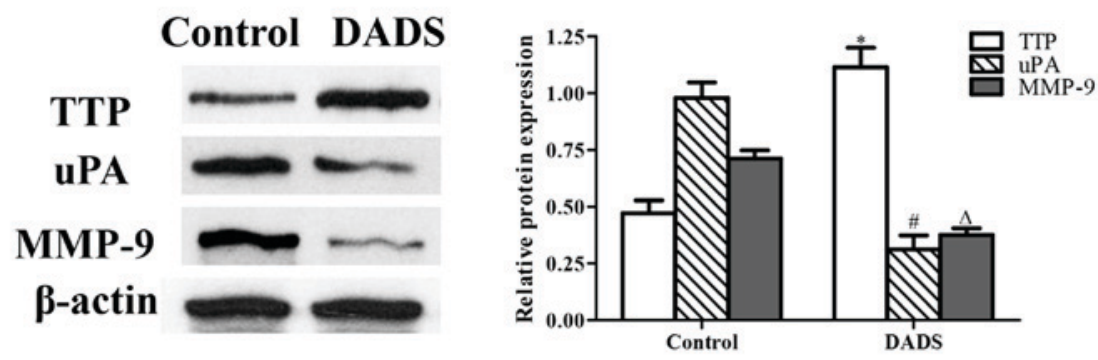

B
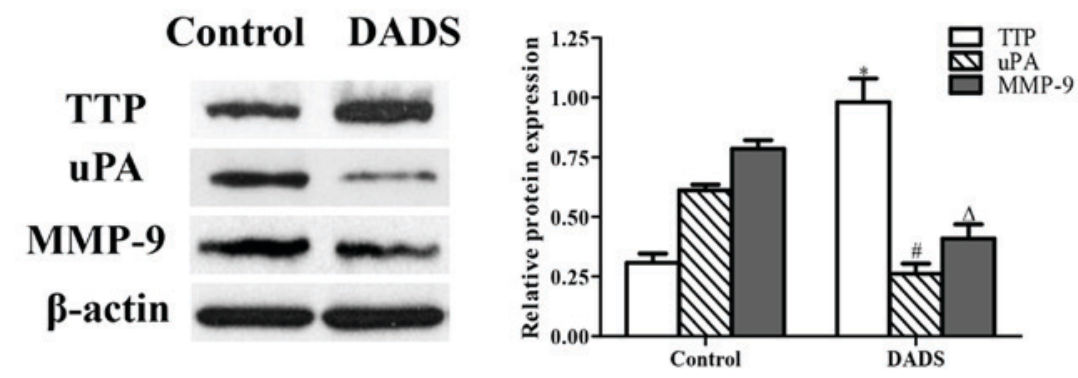

C

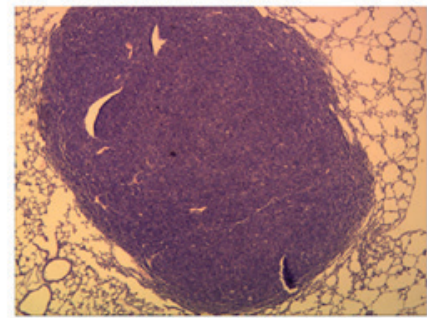

Control

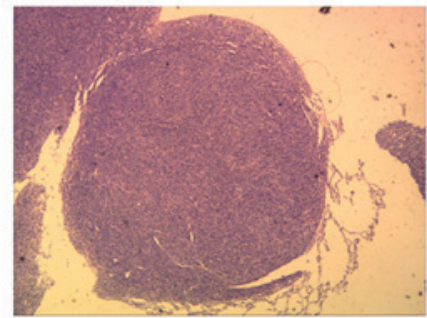

DADS

MCF-7 cells

Figure 3. Effect of DADS in the tumor of breast cancer xenografts. (A) Protein expression of TTP, uPA and MMP in (A) MCF-7 and (B) MDA-MB-231 cell xenografts. (C) Hematoxylin and eosin staining in MCF-7 cell xenografts, Magnification, $\mathrm{x} 40$. Data are presented as the mean \pm standard deviation. ${ }^{*} \Delta \mathrm{P}<0.05$ vs. corresponding control; $n=3$. DADS, diallyl disulfide; MMP, matrix metalloproteinase; uPA, urokinase-type plasminogen activator; TTP, tristetraprolin. 
A

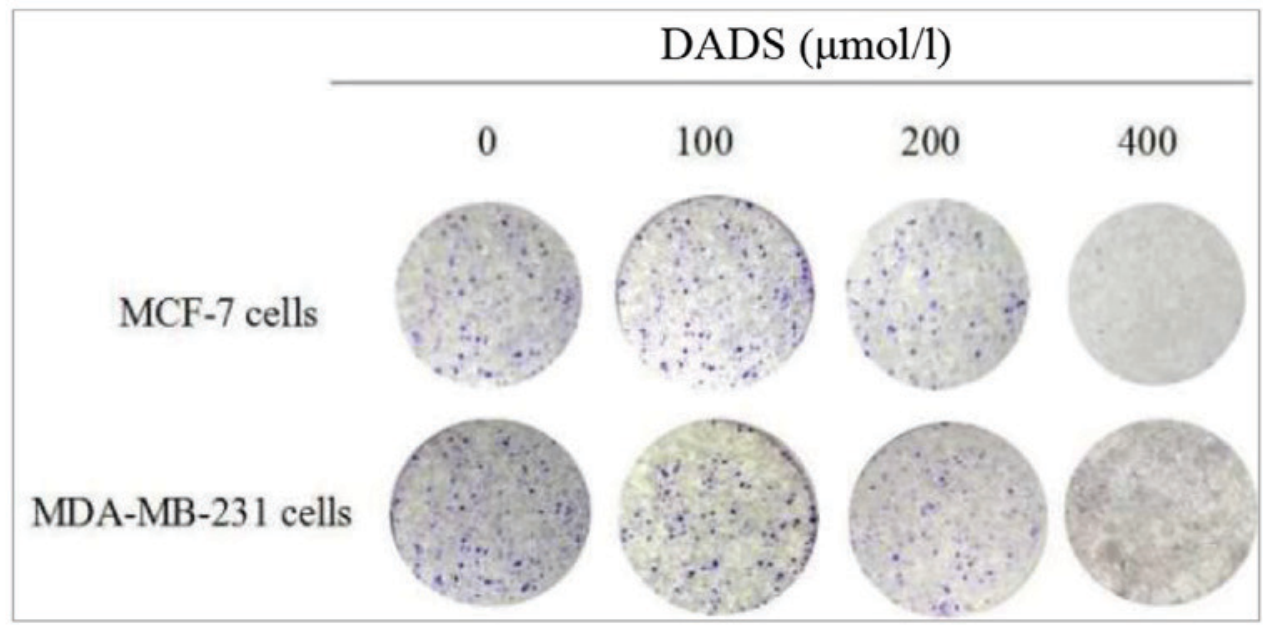

B

MCF-7 cells
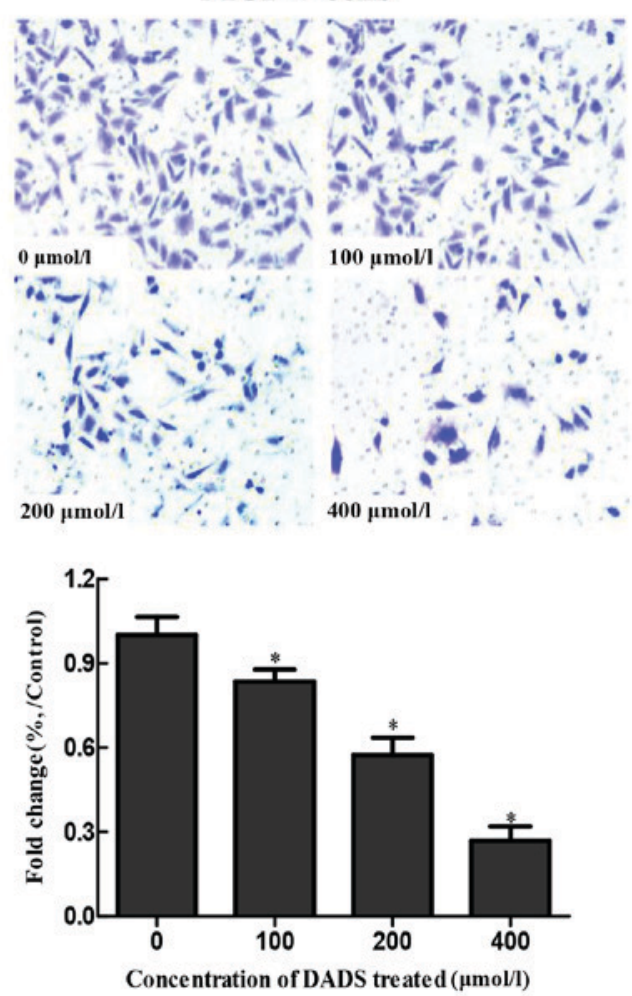

MDA-MB-231 cells
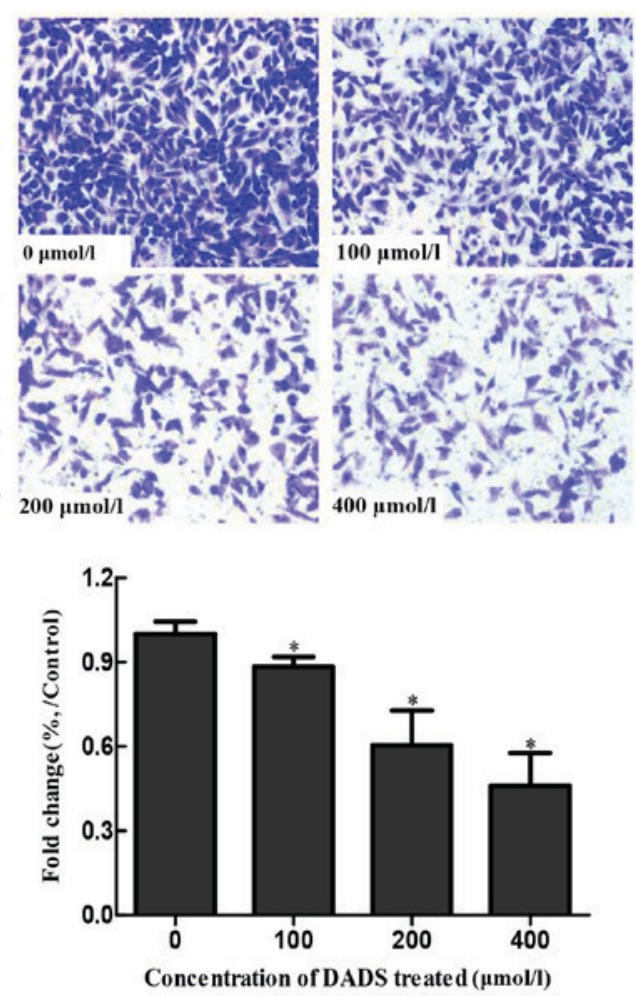

Figure 4. DADS inhibits the cloning formation and invasion of MCF-7 and MDA-MB-231 cells in a dose-dependent manner. (A) Tablet cloning assay. (B) Transwell invasion assay (magnification, $\mathrm{x} 100$ ). Data are presented as the mean \pm standard deviation. ${ }^{*} \mathrm{P}<0.05$ vs. control; $\mathrm{n}=3$. DADS, diallyl disulfide.

Using the data from the GEO dataset normal breast (17 samples) vs. breast cancer (104 samples) were analyzed. $\mathrm{P}<0.05$ was considered to indicate a statistically significant difference.

Statistical analysis. Statistical analysis was performed using SPSS software (version 20.0; IBM Corp., Armonk, NY, USA). Each experiment was repeated three times. All results are presented as the mean \pm standard deviation or standard error of three independent experiments. The $\chi^{2}$ test was applied for enumeration data. Student's t-test (unpaired) and one-way analysis of variance was used to identify statistically significant differences followed by the Student-Newman-Keuls test for further group comparison. $\mathrm{P}<0.05$ was considered to indicate a statistically significant difference.

\section{Results}

TTP is downregulated, whereas $\mathrm{APA}$ and MMP-9 are upregulated in human breast cancer compared with normal breast in the GEO database. In breast cancer microarray datasets from the GEO database, a microarray dataset (GSE42568) was identified in which TTP mRNA levels were significantly downregulated in breast cancer compared with in normal breast tissue (Fig. 1A). In the same microarray dataset, uPA and MMP-9 mRNA levels were identified to be significantly 


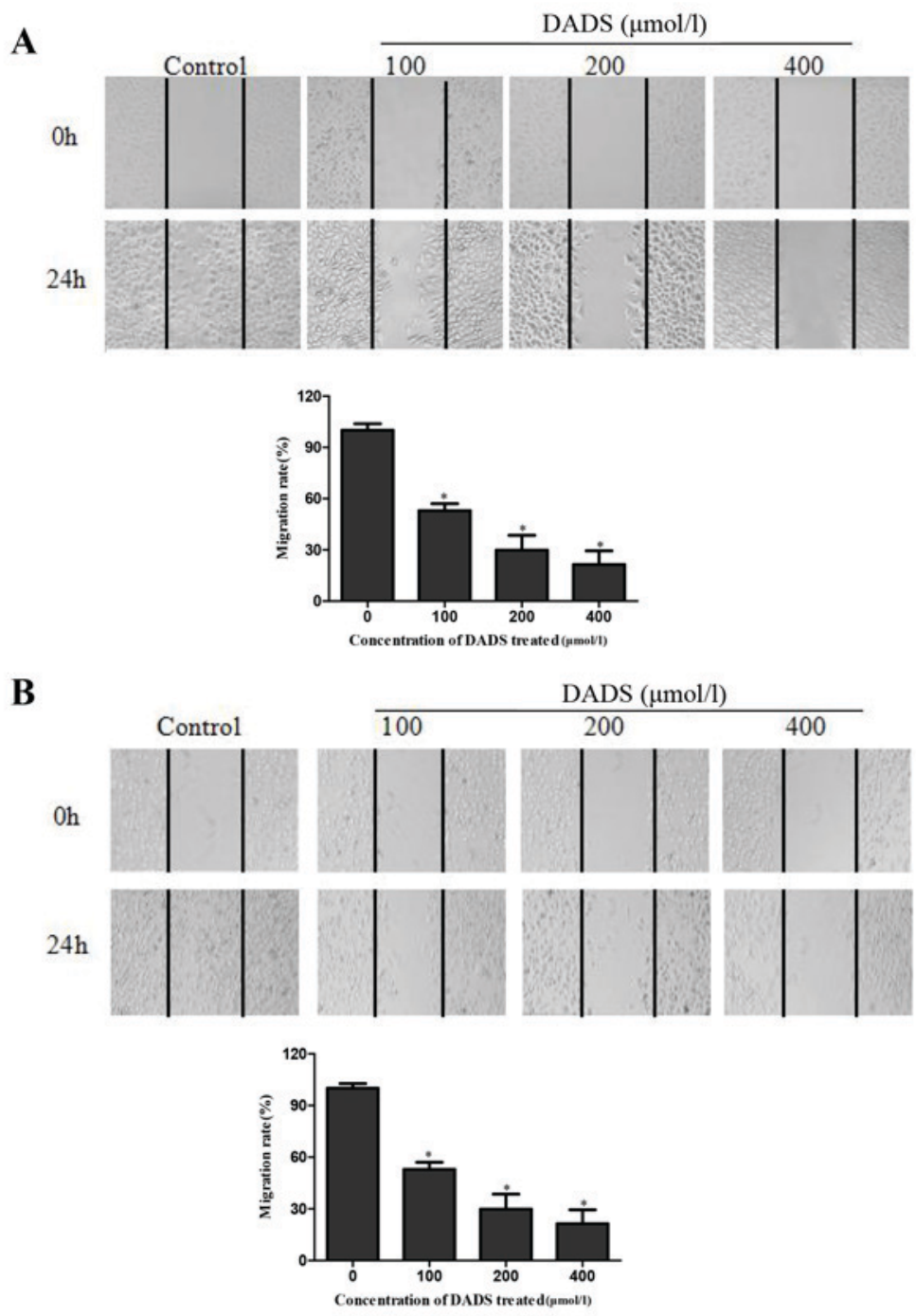

Figure 5. Effect of DADS inhibits migration as revealed using a wound healing assay in breast cancer cells. Wound healing assay representative image and quantification of (A) MCF-7 cells. (B) MDA-MB-231 cells. Magnification, $\mathrm{x} 40$. Data are presented as the mean \pm standard deviation. ${ }^{*} \mathrm{P}<0.05$ vs. control; $\mathrm{n}=3$. DADS, diallyl disulfide.

upregulated in breast cancer compared with in normal breast tissue (Fig. 1B and C).

Effect of DADS on the progression of breast cancer in vivo. Compared with the control group, the mean volume and weight of the tumors from mice in the DADS-treated group were significantly decreased (Fig. 2). Protein was extracted from the xenografts, and western blot analysis was performed to detect the expression of UPA, MMP-9 and TTP. UPA and MMP-9 protein expression levels were significantly downregulated, whereas TTP expression was significantly upregulated in the DADS-treated group compared with the corresponding control groups, suggesting that DADS induced the expression of TTP in breast cancer xenografts (Fig. 3A and B). Furthermore, $\mathrm{H} \& \mathrm{E}$ staining revealed that MCF-7-derived tumors in the DADS-treated group were markedly smaller compared with the control group (Fig. 3C).

Effect of DADS on the proliferation, invasion and migration of breast cancer cells in vitro. To examine the effect of DADS on cellular proliferation, a tablet cloning assay was performed on MCF-7 and MDA-MB-231 cells treated with DADS. It was demonstrated that the clone formation of cells was markedly decreased in a dose-dependent manner following DADS treatment (Fig. 4A). In addition, invasion and migration rates of MCF-7 and MDA-MB-231 human breast cancer cells were significantly decreased following DADS treatment, as determined using the Transwell (Fig. 4B) and wound healing assays (Fig. 5) compared with the untreated control groups.

Effect of DADS on the expression of TTP, UPA and MMP-9 in breast cancer cells. Western blotting was used to examine the expression of TTP and proteins associated with invasion, including UPA and MMP-9 in breast cancer cells. As presented in Fig. 6, DADS treatment significantly affected the expression of TTP, uPA and MMP-9 in a dose- and time-dependent manner $(\mathrm{P}<0.05)$.

Effect of knocking down TTP on the progression of breast cancer. Following transfection of MCF-7 and MDA-MB-231 cells with TTP siRNA for $24 \mathrm{~h}$, western blot analysis revealed 
A

\section{a}

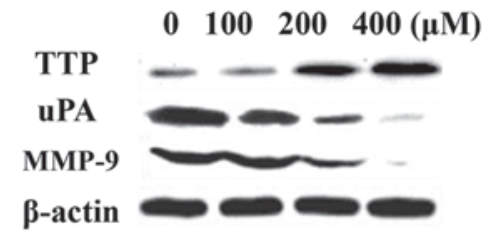

b

B

a

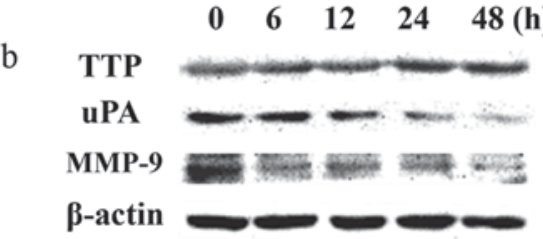

$\begin{array}{llll}0 & 100 & 200 & 400(\mu \mathrm{M})\end{array}$

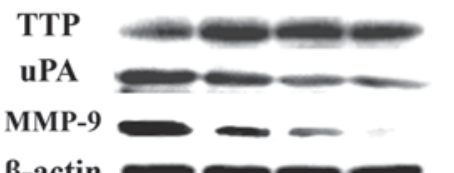

$\beta$-actin
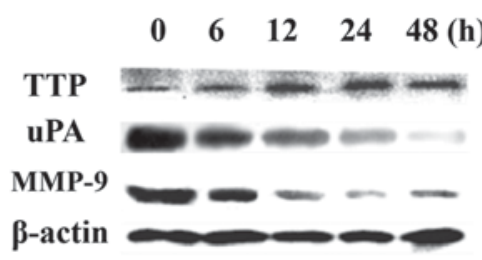
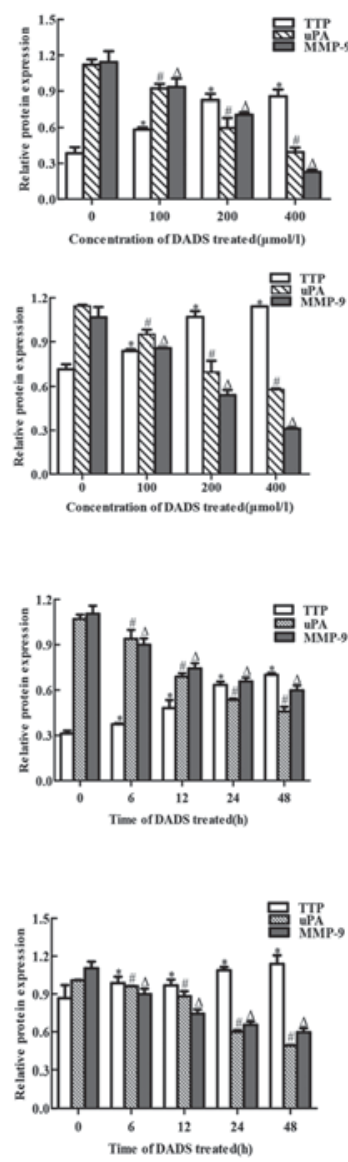

Figure 6. DADS downregulates the expression of UPA and MMP-9, and upregulates TTP expression in a dose- and time-dependent manner in breast cancer cells. Western blot analysis of (A) cells treated with different concentrations of DADS (0, 100, 200 and $400 \mu \mathrm{M})$ for $24 \mathrm{~h}$ and (B) cells treated with $200 \mu$ mol/1 DADS for different periods of time $\left(0,6,12,24\right.$ and $48 \mathrm{~h}$ ). (a) MCF-7 cells. (b) MDA-MB-231 cells. ${ }^{*} \mathrm{P}$ P $<0.05 \mathrm{vs}$. $0 \mu \mathrm{mol} / 1 \mathrm{DADS}$ or $0 \mathrm{~h}$ group; $\mathrm{n}=3$. Data are presented as the mean \pm standard deviation. DADS, diallyl disulfide; MMP, matrix metalloproteinase; uPA, urokinase-type plasminogen activator; TTP, tristetraprolin.

that TTP siRNA significantly decreased the effect that DADS exhibited on TTP, uPA and MMP-9 expression (Fig. 7). The Transwell assay demonstrated that TTP siRNA significantly reversed the anti-invasive effect of DADS in breast cancer cells (Fig. 8). Furthermore, the wound healing assay revealed that TTP siRNA counteracted the suppressive effect of DADS on breast cancer cell migration (Fig. 9). All the aforementioned results indicate that DADS may inhibit the proliferation, invasion, and migration of breast cancer cells by upregulating TTP while downregulating the expression of uPA and MMP9 (Fig. 10) and TTP may be a novel target of DADS in the progress of breast cancer.

\section{Discussion}

Breast cancer is the most commonly diagnosed type of cancer and the primary cause of cancer-associated mortalities in women worldwide (20). Over the last two decades, with the development of early diagnostic methods and more effective treatments, the mortality rate of patients with breast cancer has declined (21). However, metastatic breast cancer still lacks effective treatment and remains the primary cause of breast cancer-associated mortality (3). Therefore, full understanding the underlying mechanism of metastasis is required in order to establish methods that are able to effectively inhibit breast cancer invasion and metastasis.

DADS, one of the major organosulfur compounds in garlic, exhibits a wide variety of biological activities in vivo and exhibits promising effects as a therapeutic agent in various types of cancer. Certain anticancer mechanisms include inhibiting the production of reactive oxygen species, inhibiting cell cycle arrest and inducing apoptosis $(4,22,23)$. However, the mechanism of action of DADS in breast cancer remains unknown. Therefore, further study of the function of DADS and how it inhibits the progress of breast cancer cells is required.

In the present study, the antitumor activity of DADS in breast cancer was evaluated using in vivo and in vitro models. A mouse xenograft in vivo model was used, which demonstrated that mice treated with DADS exhibited tumors with significantly smaller weights and volumes compared with those in the control group. Furthermore, DADS treatment significantly inhibited the growth of breast cancer tumor xenografts by upregulating TTP protein expression. Using tablet cloning, Transwell and wound healing assays, it was revealed that DADS significantly inhibited the cloning formation, invasion and migration of MCF-7 and MDA-MB-231 cells compared with untreated cells. 

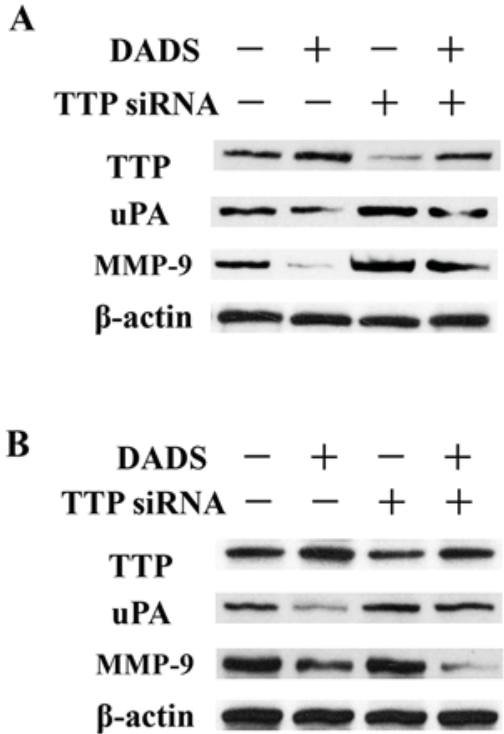
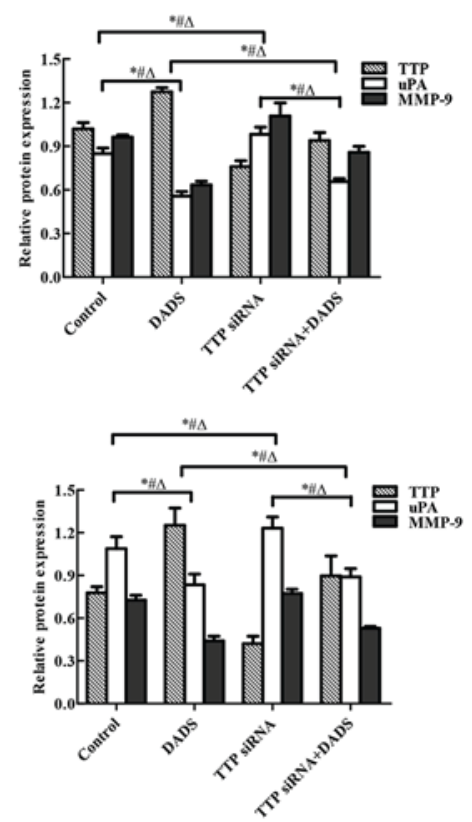

Figure 7. TTP siRNA significantly reverses the effect of DADS on the expression of TTP, uPA and MMP-9 in breast cancer cells. Western blot analysis of (A) MCF-7 and (B) MDA-MB-231 cells. ${ }^{* \#} \triangle \mathrm{P}<0.05 ; \mathrm{n}=3$. Data are presented as the mean \pm standard deviation. DADS, diallyl disulfide; MMP, matrix metalloproteinase; uPA, urokinase-type plasminogen activator; TTP, tristetraprolin; siRNA, small interfering RNA.
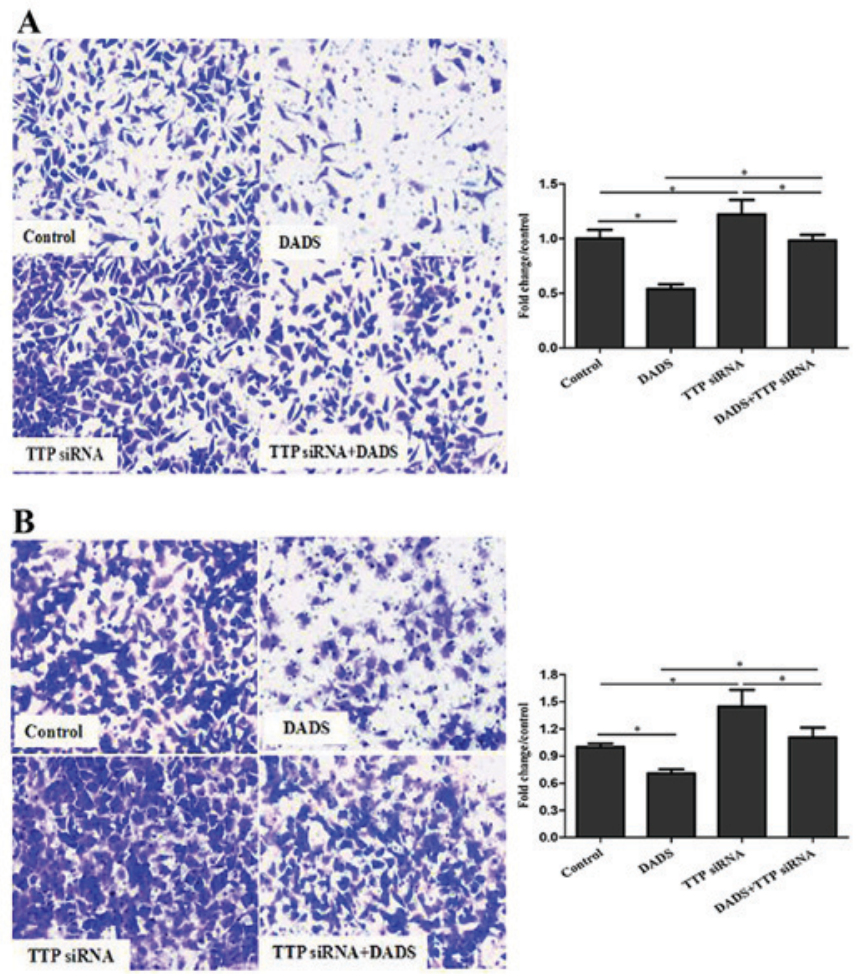

Figure 8. TTP siRNA significantly reverses the effect of DADS inhibiting the invasion in breast cancer cells. Transwell invasion assay representative image and quantification in (A) MCF-7 and (B) MDA-MB-231 cells. Magnification, $\mathrm{x} 100 .{ }^{*} \mathrm{P}<0.05 ; \mathrm{n}=3$. Data are presented as the mean \pm standard deviation. DADS, diallyl disulfide; TTP, tristetraprolin; siRNA, small interfering RNA.

Cell invasion and metastasis are two important biological characteristics of malignant tumor cells, but the degradation of the extracellular matrix occurs first. As the tumor prepares to metastasize, uPA protease is overexpressed in numerous cancer cells, including in breast carcinoma, where it serves an essential function in the metastatic process (24). uPA activates a cascade of proteases by binding to its receptor, uPA receptor (UPAR), which ultimately leads to the degradation of uPAR (25). MMPs degrade proteins that regulate various cellular behaviors associated with cancer cell differentiation, migration and invasion due to their proteolytic nature. MMP-9 serves a key function in facilitating tumor metastasis by degrading the extracellular matrix around the vicinity of the tumor and destroying the basement membrane integrity, leading to local invasion and distant metastasis of tumor cells (26). Previous research has demonstrated that uPA and MMP-9 are associated with the regulation of invasion and metastasis in various types of malignant tumor besides breast cancer, including prostate and colon cancer (27-29). The results of the present study revealed the function of DADS in inhibiting the progression of breast cancer cells.

TTP, a tandem CCCH zinc-finger RNA-binding protein, regulates the stability of several ARE-containing mRNAs. TTP serves a fundamental function in various physiological and pathological processes, including differentiation, reprogramming, metabolism, proliferation, pluripotency and tumorigenesis (30). The loss of TTP has been reported in several types of human cancer and is associated with poor prognosis (30). Previous studies identified that TTP expression is suppressed in numerous cancer cells compared with in normal cell types (31). In addition, a lack of TTP is associated with a variety of cancer-associated processes. The regulation of TTP expression has been identified to serve a function in several cancer types, including colon and breast cancer $(32,33)$. However, its function in breast cancer has not been fully investigated. TTP binds to the AREs within the 3'-untranslated regions of UPA and UPAR, and the overexpression of TTP decreases the expression of UPA and UPAR by enhancing the 
A

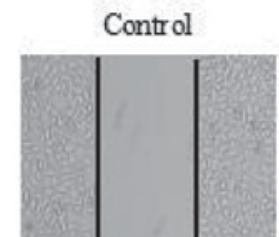

DADS

TTP siRNA

DADS+TTP siRNA

Oh
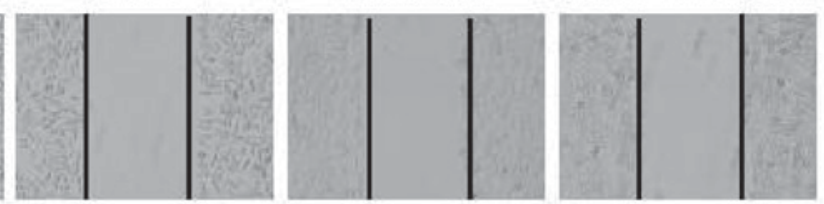

$24 \mathrm{~h}$
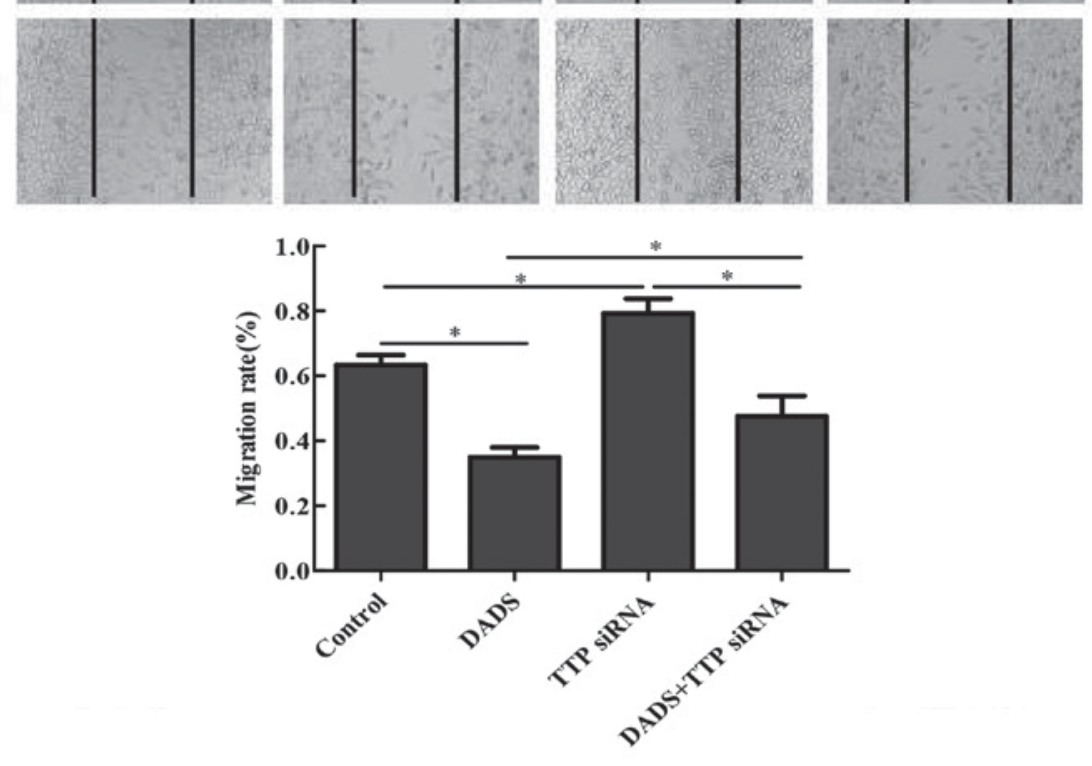

B

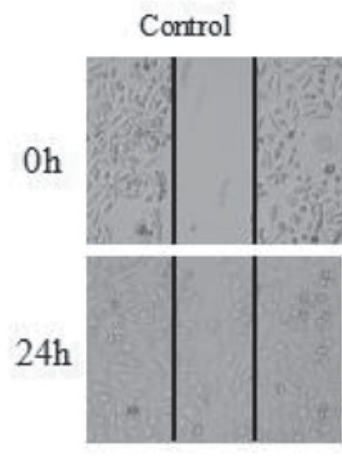

DADS
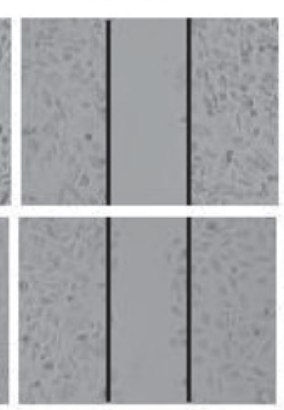

TTP siRNA
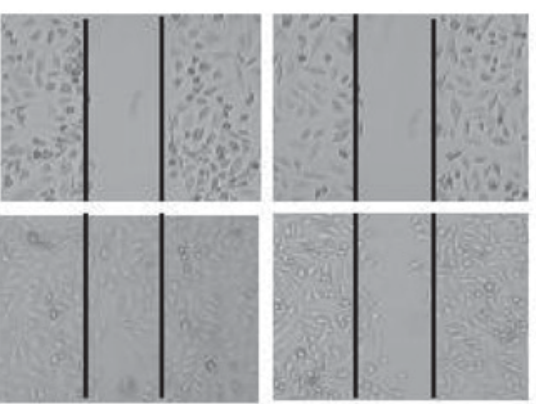

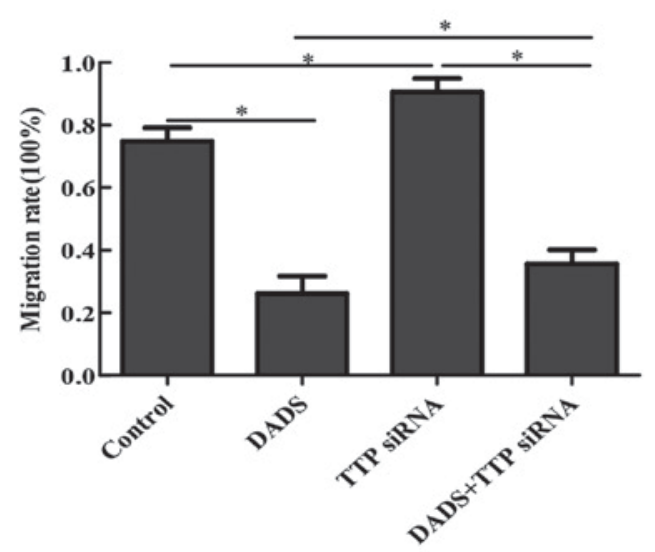

Figure 9. TTP siRNA significantly reverse the effect of DADS inhibiting the migration in breast cancer cells. Wound healing assay representative image and quantification in (A) MCF-7 and (B) MDA-MB-231 cells. Magnification, $\mathrm{x} 40 .{ }^{*} \mathrm{P}<0.05 ; \mathrm{n}=3$. Data are presented as the mean \pm standard deviation. DADS, diallyl disulfide; TTP, tristetraprolin; siRNA, small interfering RNA.

degradation of their mRNAs (34). A previous study suggested that TTP may be used as a promising therapeutic target to treat human glioma (34). A previous study identified that TTP regulates the degradation of growth factors and proto-oncogenes (16). In addition, a variety of inflammatory factors are able to enhance the invasion and metastasis of tumor cells, including tumor necrosis factor (TNF)- $\alpha$, interleukin (IL)-1 $\beta$ and IL-6. TTP is known to contribute to cytokine homeostasis by facilitating the degradation of TNF- $\alpha$ mRNA, which is essential for reducing inflammation (35). 


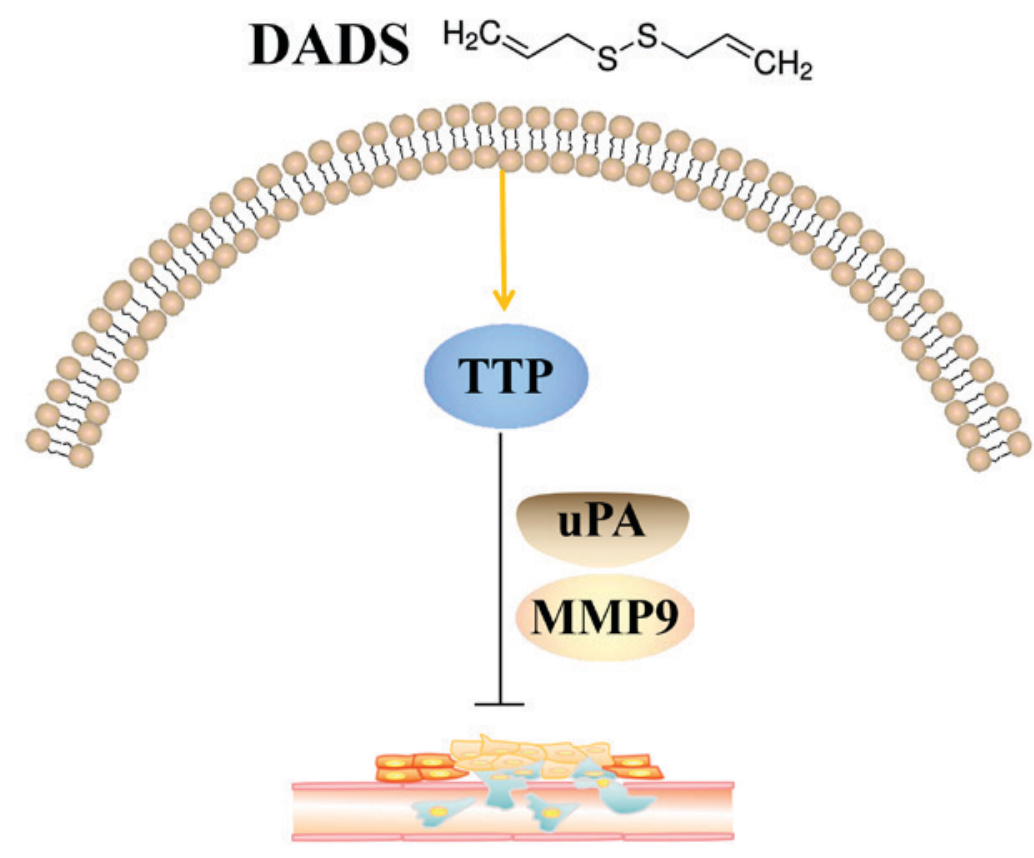

Figure 10. Schematic depiction of the mechanism underlying DADS inhibiting the progression of breast cancer. DADS may inhibit the proliferation, invasion, and migration of breast cancer cells by upregulating TTP while downregulating the expression of uPA and MMP9. DADS, diallyl disulfide; MMP, matrix metalloproteinase; uPA, urokinase-type plasminogen activator; TTP, tristetraprolin.

TTP is a molecular marker of breast cancer that regulates the proliferation, invasion and metastasis of breast cancer cells (36). In the present study, it was demonstrated that, following treatment with DADS, the protein expression of TTP was significantly upregulated, but uPA and MMP-9 expression levels were downregulated in a dose- and time-dependent manner in MCF-7 and MDA-MB-231 cells. Furthermore, silencing the expression of TTP using siRNA significantly increased the expression of UPA and MMP-9 protein, and also significantly enhanced the invasive and migratory capabilities of MCF-7 and MDA-MB-231 cells. These results suggested that TTP may be a novel target of DADS in inhibiting the progression of breast cancer. However, there remains a lack of evidence that TTP is a target of DADS, which are to be addressed in our further studies. This prompts further investigation into the function of DADS in uncontrollable inflammation and tumor growth. In conclusion, the results of the present study suggest that DADS regulates TTP expression and is a promising therapeutic target for breast cancer. Further studies on TTP are required to provide a novel basis for the targeted therapy of breast cancer, and to develop safe and effective anticancer drugs.

\section{Acknowledgements}

Not applicable.

\section{Funding}

National Natural Science Foundation of China (grant no. 81541163); the Open Fund Based on Innovation Platform of Hunan Provincial Education Department (grant no. 15K111); High-level talent research start-up fund of University of South China in 2017 (grant no. 24), Projects for Science Research of Hunan Provincial Education Department (grant no. 16C1394); Hunan Provincial Bureau of Traditional Chinese Medicine (grant no. 2014144); Hunan Provincial Cooperative Innovation Center for Molecular Target New Drug Study (grant no. 2014405); Innovation Program of College in Cooperative Innovation Center for Molecular Target New Drug of University of South China (grant no. 0223-0002-00028) and the College Student's Innovative Project of University of South China (grant no. 2016-133).

\section{Availability of data and materials}

The datasets used and/or analyzed during the current study are available from the corresponding author on reasonable request.

\section{Authors' contributions}

TX and XWL designed the study and wrote the paper; XLH, XFX and WQX performed the experiments; SJZ and JT provided reagents and mice, gave technical support and conceptual advice, and reviewed and edited the manuscript. All authors read and approved the manuscript.

\section{Ethics approval and consent to participate}

All experimental procedures conformed to the Guide for the Care and Use of Laboratory Animals published by the US National Institutes of Health (NIH Publication No. 85-23, revised 1996) and was approved by the Institutional Animal Ethics Committee of University of South China.

\section{Consent for publication}

Not applicable. 


\section{Competing interests}

The authors declare that they have no competing interests.

\section{References}

1. Niu HY, Niu CY, Wang JH, Zhang Y and He P: Health-related quality of life in women with breast cancer: A literature-based review of psychometric properties of breast cancer-specific measures. Asian Pac J Cancer Prev 15: 3533-3536, 2014.

2. Katie Lee SY and Knobf MT: Primary breast cancer decision-making among Chinese American Women: Satisfaction, regret. Nurs Res 64: 391-401, 2015.

3. Shao S, Zhao X, Zhang X, Luo M, Zuo X, Huang S, Wang Y, Gu S and Zhao X: Notch1 signaling regulates the epithelial-mesenchymal transition and invasion of breast cancer in a Slug-dependent manner. Mol Cancer 14: 28, 2015.

4. Xiao X, Chen B, Liu X, Liu P, Zheng G, Ye F, Tang H and Xie X Diallyl disulfide suppresses SRC/Ras/ERK signaling-mediated proliferation and metastasis in human breast cancer by up-regulating miR-34a. PLoS One 9: e112720, 2014.

5. Su B, Su J, Zeng Y, Liu F, Xia H, Ma YH, Zhou ZG, Zhang S Yang BM, Wu YH, et al: Diallyl disulfide suppresses epithelial-mesenchymal transition, invasion and proliferation by downregulation of LIMK1 in gastric cancer. Oncotarget 7 : 10498-10512, 2016

6. Lai KC, Hsu SC, Kuo CL, Yang JS, Ma CY, Lu HF, Tang NY, Hsia TC, Ho HC and Chung JG: Diallyl sulfide, diallyl disulfide, and diallyl trisulfide inhibit migration and invasion in human colon cancer colo 205 cells through the inhibition of matrix metalloproteinase-2, -7, and -9 expressions. Environ Toxicol 28 : 479-488, 2013

7. Luo N, Zhao LC, Shi QQ, Feng ZQ, Chen DL and Li J: Induction of apoptosis in human leukemic cell lines by diallyl disulfide via modulation of EGFR/ERK/PKM2 signaling pathways. Asian Pac J Cancer Prev 16: 3509-3515, 2015.

8. Huang J, Yang B, Xiang T, Peng W, Qiu Z, Wan J, Zhang L, Li H, $\mathrm{Li} \mathrm{H}$ and Ren G: Diallyl disulfide inhibits growth and metastatic potential of human triple-negative breast cancer cells through inactivation of the $\beta$-catenin signaling pathway. Mol Nutr Food Res 59: 1063-1075, 2015.

9. Ciocci M, Iorio E, Carotenuto F, Khashoggi HA, Nanni F and Melino S: H2S-releasing nanoemulsions: A new formulation to inhibit tumor cells proliferation and improve tissue repair. Oncotarget 7: 84338-84358, 2016.

10. Yi L, Shan J, Chen X, Li G, Li L, Tan H and Su Q: Involvement of calreticulin in cell proliferation, invasion and differentiation in diallyl disulfide-treated HL-60 cells. Oncol Lett 12: 1861-1867, 2016.

11. Su B, Su J, He H, Wu Y, Xia H, Zeng X, Dai W, Ai X, Ling H, Jiang $\mathrm{H}$ and $\mathrm{Su}$ Q: Identification of potential targets for diallyl disulfide in human gastric cancer MGC-803 cells using proteomics approaches. Oncol Rep 33: 2484-2494, 2015.

12. Sun Y, Wang X, Zhou Q, Lu Y, Zhang H, Chen Q, Zhao M and Su S: Inhibitory effect of emodin on migration, invasion and metastasis of human breast cancer MDA-MB-231 cells in vitro and in vivo. Oncol Rep 33: 338-346, 2015.

13. Jacob A and Prekeris R: The regulation of MMP targeting to invadopodia during cancer metastasis. Front Cell Dev Biol 3: 4, 2015.

14. Lampelj M, Arko D, Cas-Sikosek N, Kavalar R, Ravnik M, Jezersek-Novakovic B, Dobnik S, Dovnik NF and Takac I: Urokinase plasminogen activator (uPA) and plasminogen activator inhibitor type-1 (PAI-1) in breast cancer-correlation with traditional prognostic factors. Radiol Oncol 49: 357-364, 2015.

15. Melanson BD, Bose R, Hamill JD, Marcellus KA, Pan EF and McKay BC: The role of mRNA decay in p53 induced gene expression. RNA 17: 2222-2234, 2011.

16. Brooks SA and Blackshear PJ: Tristetraprolin (TTP): Interactions with mRNA and proteins, and current thoughts on mechanisms of action. Biochim Biophys Acta 1829: 666-679, 2013

17. Barrios-García T, Tecalco-Cruz A, Gómez-Romero V, Reyes-Carmona S, Meneses-Morales I and León-Del-Río A: Tristetraprolin represses estrogen receptor $\alpha$ transactivation in breast cancer cells. J Biol Chem 289: 15554-15565, 2014
18. Tran DDH, Koch A, Allister A, Saran S, Ewald F, Koch M, Nashan B and Tamura T: Treatment with MAPKAP2 (MK2) inhibitor and DNA methylation inhibitor, 5-aza dC, synergistically triggers apoptosis in hepatocellular carcinoma (HCC) via tristetraprolin (TTP). Cell Signal 28: 1872-1880, 2016.

19. Van Tubergen EA, Banerjee R, Liu M, Vander Broek R, Light E, Kuo S, Feinberg SE, Willis AL, Wolf G, Carey T, et al: Inactivation or loss of TTP promotes invasion in head and neck cancer via transcript stabilization and secretion of MMP9, MMP2, and IL-6. Clin Cancer Res 19: 1169-1179, 2013.

20. Redig AJ and McAllister SS: Breast cancer as a systemic disease: A view of metastasis. J Intern Med 274: 113-126, 2013

21. Warrier S, Tapia G, Goltsman D and Beith J: An update in breast cancer screening and management. Womens Health (Lond) 12: 229-239, 2016.

22. Lei XY, Yao SQ, Zu XY, Huang ZX, Liu LJ, Zhong M, Zhu BY, Tang SS and Liao DF: Apoptosis induced by diallyl disulfide in human breast cancer cell line MCF-7. Acta Pharmacol Sin 29: $1233-1239,2008$

23. Wang G, Liu G, Ye Y, Fu Y and Zhang X: Upregulation of miR-34a by diallyl disulfide suppresses invasion and induces apoptosis in SGC-7901 cells through inhibition of the PI3K/Akt signaling pathway. Oncol Lett 11: 2661-2667, 2016.

24. Pei S, Yang X, Wang H, Zhang H, Zhou B, Zhang D and Lin D: Plantamajoside, a potential anti-tumor herbal medicine inhibits breast cancer growth and pulmonary metastasis by decreasing the activity of matrix metalloproteinase- 9 and -2 . BMC Cancer 15 : $965,2015$.

25. Noh H, Hong S and Huang S: Role of urokinase receptor in tumor progression and development. Theranostics 3: 487-495, 2013.

26. Page-McCaw A, Ewald AJ and Werb Z: Matrix metalloproteinases and the regulation of tissue remodelling. Nat Rev Mol Cell Biol 8: 221-233, 2007.

27. Moirangthem A, Bondhopadhyay B, Mukherjee M, Bandyopadhyay A, Mukherjee N, Konar K, Bhattacharya S and Basu A: Simultaneous knockdown of uPA and MMP9 can reduce breast cancer progression by increasing cell-cell adhesion and modulating EMT genes. Sci Rep 6: 21903, 2016.

28. Shi C, Zhang N, Feng Y, Cao J, Chen X and Liu B: Aspirin inhibits IKK- $\beta$-mediated prostate cancer cell invasion by targeting matrix Metalloproteinase-9 and Urokinase-Type plasminogen activator. Cell Physiol Biochem 41: 1313-1324, 2017.

29. Tong W, Wang Q, Sun D and Suo J: Curcumin suppresses colon cancer cell invasion via AMPK-induced inhibition of NF- $\kappa$ B, uPA activator and MMP9. Oncol Lett 12: 4139-4146, 2016.

30. Guo J, Qu H, Chen Y and Xia J: The role of RNA-binding protein tristetraprolin in cancer and immunity. Med Oncol 34: 196, 2017.

31. GriseriP,Bourcier C,Hieblot C,Essafi-Benkhadir K, Chamorey E, Touriol $\mathrm{C}$ and Pagès $\mathrm{G}$ : A synonymous polymorphism of the Tristetraprolin (TTP) gene, an AU-rich mRNA-binding protein, affects translation efficiency and response to Herceptin treatment in breast cancer patients. Hum Mol Genet 20: 4556-4568, 2011.

32. Sobolewski C, Sanduja S, Blanco FF, Hu L and Dixon DA Histone deacetylase inhibitors activate tristetraprolin expression through induction of early growth response protein 1 (EGR1) in colorectal cancer cells. Biomolecules 5: 2035-2055, 2015.

33. Pandiri I, Chen Y, Joe Y, Kim HJ, Park J, Chung HT and Park JW: Tristetraprolin mediates the anti-proliferative effects of metformin in breast cancer cells. Breast Cancer Res Treat 156: 57-64, 2016.

34. Ryu J, Yoon NA, Lee YK, Jeong JY, Kang S, Seong H, Choi J, Park N, Kim N, Cho WJ, et al: Tristetraprolin inhibits the growth of human glioma cells through downregulation of urokinase plasminogen activator/urokinase plasminogen activator receptor mRNAs. Mol Cells 38: 156-162, 2015.

35. Prabhala P and Ammit AJ: Tristetraprolin and its role in regulation of airway inflammation. Mol Pharmacol 87: 629-638, 2015.

36. Gebeshuber CA,Zatloukal K and Martinez J: miR-29a suppresses tristetraprolin, which is a regulator of epithelial polarity and metastasis. EMBO Rep 10: 400-405, 2009.

This work is licensed under a Creative Commons Attribution-NonCommercial-NoDerivatives 4.0 International (CC BY-NC-ND 4.0) License. 